\title{
Tecnura
}

INVESTIGACIÓN

\section{Control, supervisión y representación matemática de un proceso de biodigestión anaerobia para la biomasa contenido ruminal bovino}

\section{Control, supervision, and mathematical representation of an anaerobic digestion process for biomass bovine ruminal content}

\author{
Álvaro Ervey Pulido Aponte1, Hernán Mauricio Rivera Escobar², Jhon Jairo Espitia Pardo ${ }^{3}$
}

Fecha de recepción: 22 de marzo de 2018

Fecha de aceptación: 30 de agosto de 2018

Cómo citar: Pulido A., A.E., Rivera E., H.M. y Espitia P., J.J. (2018). Control, supervisión y representación matemática de un proceso de biodigestion anaerobia para la biomasa contenido ruminal bovino. Tecnura, 22(58), 37-52. DOI: https://doi.org/10.14483/22487638.10965

\section{Resumen}

Contexto: En Colombia, el contenido rumial bovino (CRB) es vertido generalmente a los cauces de agua y campos a cielo abierto y, dada su alta carga orgánica, su disposición final representa una amenaza de tipo ambiental, económica y social. A la fecha, es limitada la implementación de tecnologías que permitan reducir el impacto debido en parte a la escasa caracterización del CRB, información que es requerida para la construcción de modelos matemáticos de digestión anaerobia que expliquen la reducción de este bio-sólido; por otro lado, la restringida aplicación de sistemas de control y supervisión enfocados a la generación de subproductos con potencial aprovechamiento energético también constituye un motivo.

Objetivo: Documentar los métodos utilizados en el desarrollo de un sistema de control y supervisión de temperatura y presión para un biodigestor discontinuo a escala de laboratorio con biomasa CRB.

Métodos: Se realizó la implementación de un sistema de adquisición distribuida de señales y una interfaz de servidor web; se identificó un modelo dinámico correspondiente al comportamiento del sistema de digestión anaerobia para esta biomasa CRB.
Resultados: Se logró caracterizar, mediante gravimetría y calorimetría, variables asociadas al sistema de digestión anaerobia con CRB. Frente al control, el controlador on-off mantuvo la presión del gas en el rango deseado y el controlador PI garantizó la temperatura deseada del proceso.

Conclusión: Fue posible generar biogás a partir de biomasa CRB desde esquemas básicos de caracterización, supervisión y control del proceso.

Palabras clave: biorreactor, temperatura, presión, biomasa, control, supervisión.

\begin{abstract}
Introduction: In Colombia, bovine ruminal content (BRC) is generally poured into water courses and open fields and, given its high organic load, its final disposal represents an environmental, economic, and social threat. To date, the implementation of technologies that reduce the impact is limited. This is due, on the one hand, to the scarce characterization of the BRC, which is required for the construction of mathematical models of anaerobic digestion that explain the reduction of this bio-solid; and on the other hand, to the restricted application of control
\end{abstract}

1 Ingeniero en control, candidato a magíster en Ingeniería de la Universidad Nacional de Colombia. Bogotá, Colombia. Contacto: apulidoa@unal.edu.co

2 Licenciado en Química, especialista en Bioquímica, magíster en Bioquímica, candidato a doctor en Ciencias Biomédicas de la Universidad Nacional de Colombia. Bogotá, Colombia. Contacto: hmriverae@unal.edu.co

3 Ingeniero en Control. Universidad Distrital Francisco José de Caldas. Bogotá, Colombia. 
and supervision systems focused on the generation of by-products with potential use.

Aim: To document the methods used in the development of control and monitoring system ot temperature and pressure, for a discontinuous bio-digester at laboratory scale with biomass BRC.

Methods: The implementation of a distributed signal acquisition system and a web server interface were carried out; additionally, a dynamic model corresponding to the behavior of the anaerobic digestion system for this BRC biomass was identified.
Results: Using gravimetry and calorimetry, it was possible to characterize variables associated with the anaerobic digestion system with BRC. As for the control, the on-off controller kept the gas pressure in the desired range and the PI controller guaranteed the desired temperature of the process.

Conclusion: It was possible to generate biogas from biomass BRC from basic schemes of characterization, supervision, and control of the process.

Keywords: bioreactor, temperature, pressure, biomass, control, monitoring.

\section{INTRODUCCIÓN}

El contenido ruminal bovino (CRB) es un material predigerido localizado en el primer estómago del ganado bovino o vacuno y por ende, un subproducto originado de su sacrificio (figura 1A) (Ríos y Ramírez, 2012). Debido a su riqueza en composición química, flora y fauna microbiana (Febres y López, 2007; Lettal et al., 2012; Pedreira et al., 2013), el CRB puede ser dispuesto como biomasa en procesos de biodigestión que generen productos con potencial energético y mitiguen las problemáticas sociales asociadas al impacto ambiental que representa el actual manejo y disposición final de este tipo de residuos (Cadavid, 2001; Flórez, Barco y Rincón, 2016; Moss, Jouany y Newbold, 2000; Ríos y Ramírez, 2012; Uicab-Brito y Castro, 2003; Vargas-Soplín y Ramírez-Candia, 2017).

La biodigestión es un proceso espontaneo y natural que puede llevarse a cabo en un espacio físico llamado biodigestor, en donde los reactivos se transforman en productos en función de variables físicas, químicas y biológicas, como, la presión del sistema, la estequiometría, la temperatura y el $\mathrm{pH}$ de la reacción, y el metabolismo de diferentes microorganismos (Fiotto, 2013; Ramírez, 2010). Durante la biodigestión que se efectúa en ausencia de oxígeno, también llamada biodigestión anaerobia, asociada a bacterias metanogénicas (Arévalo, 2015; Smith, 1968; Vincent, 1997), tiene lugar una serie de reacciones bioquímicas que transforman el material orgánico, llamado biomasa, en subproductos, como dióxido de carbono (CO2), sulfuro de hidrogeno $(\mathrm{H} 2 \mathrm{~S})$ y metano $(\mathrm{CH} 4)$ (Arévalo, 2015; Denbigh y Turner, 1990; Quiñones, 2006), este último, biogás, con un gran potencial energético.

Para efectos de aprovechamiento del potencial energético en condiciones controladas, se requiere un sistema que garantice la eficiencia de la biodigestión, definida como cantidad de biomasa transformada en biogás por unidad de tiempo (Arévalo, 2015; Bermúdez, 1988; Carrillo, 2004). Mejorar la eficiencia requiere implementar sistemas que controlen algunas de las variables ya mencionadas, como temperatura, que incide tanto en la actividad bioquímica de las bacterias metanogénicas, como en la cinética de la reacción, o la presión, asociada con garantizar la estabilidad de la operación (Gómez y Hernández, 2010; Tardáguila, 2008). Otros factores, como el funcionamiento inadecuado de la instrumentación, el cargue incorrecto de la biomasa, la presencia de agentes biológicos y químicos, generalmente representados como contaminantes en el agua, el oxígeno del aire, los residuos de detergentes u otros compuestos, también se asocian con la reducción de la eficiencia de la biodigestión (Ramírez, 2010).

En la actualidad, la actividad ganadera en Colombia es creciente, lo que garantiza una fuente permanente de CRB (Ledesma, Gallego y Peláez, 
2002), pese a ello, sigue siendo insuficiente la implementación de sistemas de control y supervisión que garanticen la eficiencia de un proceso de biodigestión anaerobia con biomasa CRB que genere productos con potencial energético (Cadavid, 2001). Empero, el uso de biodigestores para la producción de biogás ha tenido un importante auge en las últimas décadas, en parte, debido a que la tecnología que requiere este tipo de procesos existe en el mercado y su costo es muy inferior a otros implementados en la producción de energías alternativas (Álvarez, 2013; Torres y Manga, 2013).

En este artículo se describe el proceso de producción de biogás a partir de un reactor de

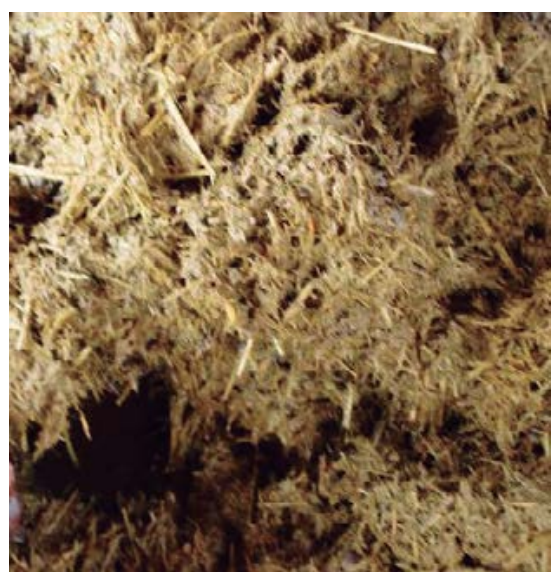

(A)

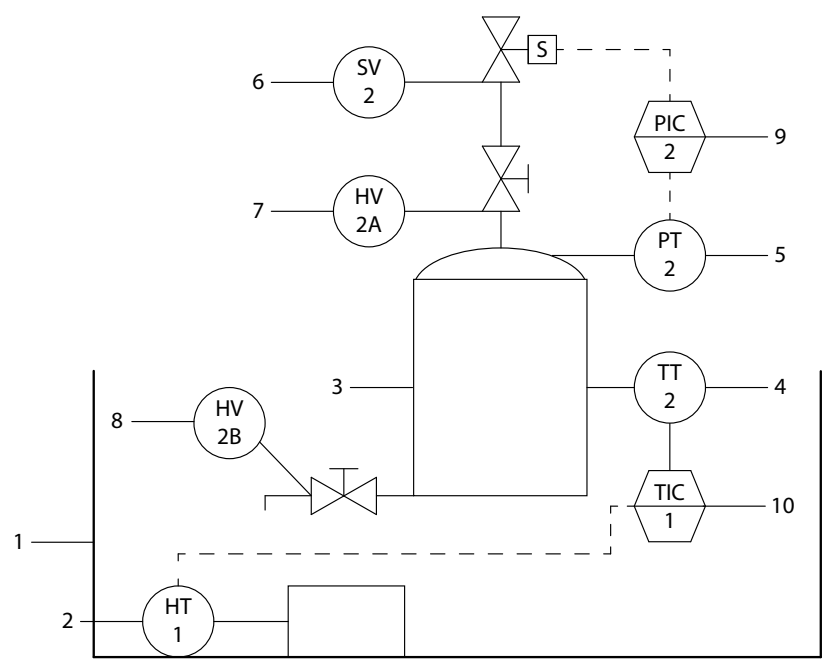

(B)

(C)

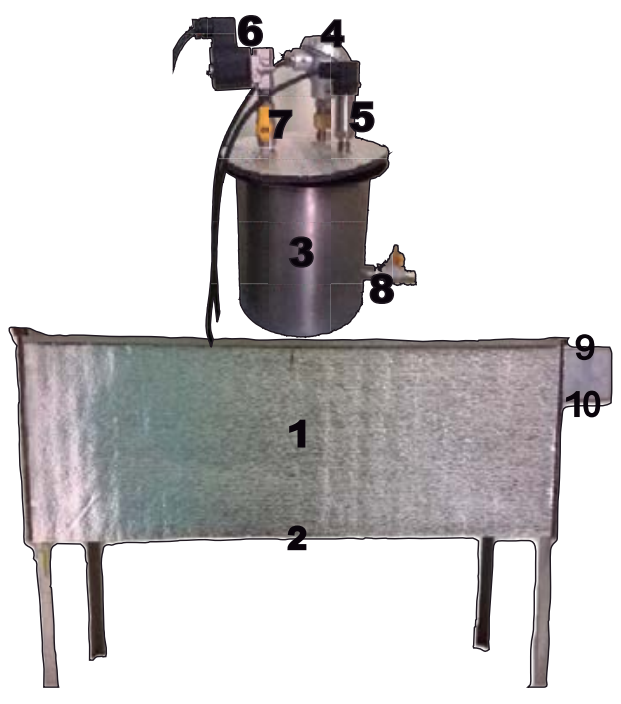

Figura 1. Características generales del sistema de digestión anaerobia con biomasa CRB. A. Fotografía representativa de la biomasa CRB. B. Diagrama P\&ID del sistema de biodigestión anaerobia implementado. C. Fotografía de B.

Fuente: elaboración propia. 
digestión anaerobia con biomasa CRB a escala de laboratorio, el cual incluye los protocolos experimentales implementados para la caracterización y cargue de la biomasa, el acondicionamiento del biorreactor mediante la implementación de instrumentación industrial y la recolección y almacenamiento del biogás. Igualmente, se documenta la implementación de estrategias básicas de control y supervisión para las variables temperatura y presión por medio de un sistema de adquisición distribuida de señales y una interfaz de servidor web; finalmente, se propone un modelo matemático basado en la transferencia de calor, composición química de la biomasa y la cuantificación del producto obtenido.

\section{METODOLOGÍA}

El diseño experimental incluyó cinco etapas: acondicionamiento del reactor, experimentación, supervisión, control y modelo dinámico.

\section{Acondicionamiento del reactor}

Se utilizó un recipiente cilíndrico de acero inoxidable de volumen 10 litros, con tapa del mismo material, ajustada herméticamente con un empaque de caucho y 8 pernos. Esta tapa presentaba tres conectores de proceso (dos de $1 / 4^{\prime \prime}$ y uno de $1 / 2^{\prime \prime}$ ), los cuales fueron adaptados a la instrumentación (figura 1C). Esta composición de elementos se asemeja a la estructura de un reactor discontinuo o Batch (Fiotto, 2013; Levenspiel y Barreiro, 2002; Smith, 1968), espacio físico donde ocurrió la digestión anaerobia de la biomasa CRB. El instrumental industrial con salida estándar de 4-20 mA, incluyó dos transmisores, uno de temperatura y uno de presión, PT100 y YB131 respectivamente. EI PT100 contenía un largo de inserción de $25 \mathrm{~cm}$ unido a un cabezal encargado de aislar la temperatura ambiente de la temperatura de proceso al interior del reactor, mientras que el YB131 es un transmisor de presión para gases y vapor. Para garantizar las condiciones de temperatura, se incorporó al biodigestor un sistema de calentamiento mediante baño maría y para aliviar la sobrepresión generada en el proceso se implementó una válvula solenoide PDI01044 a una de las conexiones de proceso, en la tabla 1 se referencian los TAG de instrumentación correspondientes a las características generales del sistema de digestión anaerobia con biomasa CRB y su respectivo diagrama P\&ID (piping and instrumentation diagram) ilustrado en la figura 1.

Tabla 1. Elementos fijados para los elementos del diagrama P\&ID

\begin{tabular}{llc}
\hline ITEM & \multicolumn{1}{c}{ DESCRIPCIÓN } & TAG \\
\hline 1 & Recipiente adiabático & N/A \\
\hline 2 & Heating Element-resistencia de calentamiento & HT 1 \\
\hline 3 & Biorreactor & N/A \\
\hline 4 & Transmisor de temperatura PT100 & TT 2 \\
\hline 5 & Transmisor de presión YB131 & PT 2 \\
\hline 6 & Válvula solenoide PDI01044 & SV 2 \\
\hline 7 & Válvula manual (1) & HV 2A \\
\hline 8 & Válvula manual (2) & HV 2B \\
\hline 9 & Control-indicador de presión & PIC 2 \\
\hline 10 & Control-indicador de temperatura & TIC 1 \\
\hline
\end{tabular}

Fuente: elaboración propia. 


\section{Experimentación}

La etapa experimental se desarrolló en el laboratorio de química básica de la Facultad Tecnológica de la Universidad Distrital Francisco José de Caldas, Bogotá D.C. El CRB fue donado por la planta de sacrificio del municipio de Nocaima (Cundinamarca) y por los frigoríficos de Guadalupe y San Martín, en Bogotá D.C., y fue extraído bajo protocolos previamente establecidos por cada uno de los establecimientos arriba mencionados. EI CRB se mantuvo sellado en un contenedor estéril de polipropileno a $7{ }^{\circ} \mathrm{C}$ en nevera hasta su uso.

\section{Caracterización de la biomasa CRB}

Por gravimetría se determinó la densidad y los porcentajes de humedad y de materia orgánica de la biomasa CRB; y por calorimetría, su calor especifico (Burghardt, 1984; Kreith, Manglik y Bohn, 2012; Torge, 1989; Vogel, 1960), estos resultados fueron parámetros necesarios para el cargue y variables del modelo dinámico del sistema.

La densidad $D$ se estableció a partir del cociente entre la masa neta Mh (ecuación (1)), y el volumen del CRB (ecuación (2)).

$$
M_{h}=M_{T}-M_{p}
$$

Donde $M_{T}$ corresponde a la suma de la masa del CRB con la masa de la probeta y $M p$ es la masa de la probeta vacía.

$$
D=\frac{M_{h}}{V}
$$

El porcentaje de humedad $H$ se estableció a partir del cociente entre Mdif y Mh, donde Mdif representa la diferencia entre $10 \mathrm{~g}$ de masa de $\mathrm{CRB}, M h$, y su masa deshidratada, $M d h$, en horno a $110{ }^{\circ} \mathrm{C}$ por una hora (ecuación (3)).

$$
H=\frac{M_{d i f}}{M_{h}} * 100 \%
$$

El porcentaje de materia orgánica, mo, se determinó a partir del cociente entre Mog y $M d h$, donde Mog representa la diferencia entre $M d h$ y su respectiva masa incinerada, $\mathrm{Mic}$, en una mufla a $600{ }^{\circ} \mathrm{C}$ por 30 minutos (ecuación (4)).

$$
m o=\frac{M_{o g}}{M_{d h}} * 100 \%
$$

Para determinar el calor especifico de la biomasa CRB, CCRB, se igualaron los flujos caloríficos del agua y de la biomasa CRB en términos del teorema fundamental de la calorimetría (ecuación (5)), donde $\mathrm{CH} 2 \mathrm{O}$ y $C \mathrm{CRB}$ corresponden al calor especifico; $m H 2 O$ y $m C R B$ la masa; y $\Delta$ $T H 2 O$ y $\triangle T C R B$ la diferencia de temperatura del agua, $\mathrm{H} 2 \mathrm{O}$, y de la biomasa $\mathrm{CRB}$ respectivamente. Dado que $m H 2 O$ y $m C R B$ fueron equivalentes y que $\mathrm{CH} 20$ es constante, se despejó $C C R B$, definido como el cociente entre $\triangle T H 20$ y $\triangle T C R B$ (ecuación (6)).

$$
\begin{gathered}
C_{\mathrm{H}_{2} \mathrm{O}} \cdot m_{\mathrm{H}_{2} \mathrm{O}} \cdot\left(\Delta T_{\mathrm{H}_{2} \mathrm{O}}\right)=C_{C R B} \cdot m_{C R B} \cdot\left(\Delta T_{C R B}\right) \\
\frac{(\Delta \mathrm{t})_{\mathrm{H}_{2} \mathrm{O}}}{(\Delta \mathrm{t})_{\mathrm{CRB}}}=C_{\mathrm{CRB}}
\end{gathered}
$$

Todos los ensayos fueron realizados por triplicado desde muestras independientes.

\section{Cargue de la biomasa CRB}

Previo al cargue, se realizó la limpieza de todo el material, incluido el biodigestor, con soluciones de hipoclorito al 0,3\% volumen, etanol $40 \%$ volumen y agua destilada. Para el cargue de la biomasa CRB, se incorporó al reactor una relación 1:1 masa del CRB y agua destilada hasta completar un $80 \%$ de la capacidad del contenedor. Posterior al cargue, se selló el biodigestor eliminando el aire contenido mediante bomba de vacío. Para garantizar un rango de temperatura en el mesófílo, entre $34{ }^{\circ} \mathrm{C}$ y $36{ }^{\circ} \mathrm{C}$, el biodigestor fue parcialmente sumergido en un sistema de calentamiento por baño maría (figura 1B, 1C). El proceso de biodigestión se llevó a cabo durante 37 días; al séptimo día, se realizó la primera extracción del biogás generado y posteriores extracciones fueron dependientes de la presión acumulada. 


\section{Supervisión}

Para establecer el sistema de supervisión del proceso, se adecuaron las señales análogas correspondientes a las variables temperatura y presión entregadas por los transmisores PT100 y YB131 respectivamente en lazos de corriente 4-20 mA; se utilizó un sistema de adquisición distribuida de señales a través de las entradas análogas multiplexadas de la tarjeta Arduino UNO, la cual digitalizó y transmitió por protocolo USB (universal serial bus) la información a un sistema embebido Raspberry pi2, donde se efectuó el procesamiento de la información a través de un script programado en lenguaje Python, la retransmisión de esta información se realizó desde un enrutador local mediante protocolos SSH y http, a una interfaz de servidor web creada mediante un framework denominado WebIOPi, donde se realizó el monitoreo y seguimiento del proceso. De forma análoga, se obtuvo acceso remoto a la consola de programación mediante el cliente SSH-PUTTYHTTP/SERVER (figura 2).

\section{Control}

El control de la temperatura y la presión del proceso se realizó mediante la adquisición y procesamiento de los valores análogos entregados por los transmisores PT100 y YB131 al sistema de adquisición distribuida de señales, Arduino UNO, en donde se programaron las acciones de control y se conmutaron los actuadores por medio de relés de estado sólido. La presión fue controlada mediante la incorporación de una válvula solenoide PDI01044 al biodigestor, no obstante, el principio del funcionamiento se restringió a la acción de un control on-off, donde se configuró el valor de set point a 9 psi con una histéresis de 0,2 psi.

Para el control de temperatura de la biomasa CRB al interior del reactor, se partió de la identificación de la planta, para ello, se utilizó la herramienta computacional (system identification toolbox del software MATLAB) en un proceso de identificación clásica (Alfaro, 2001), estableciendo un modelo matemático, en donde la señal escalón representó la señal de excitación. Como el sistema térmico ofreció monotonía creciente sin oscilaciones en su respuesta, se identificó un modelo de referencia de primer orden con retardo que describió con aproximación el comportamiento del ingenio en fase de calentamiento. Una vez determinado el modelo de identificación, se efectuó un método empírico de sintonización de los parámetros del controlador, según las reglas de

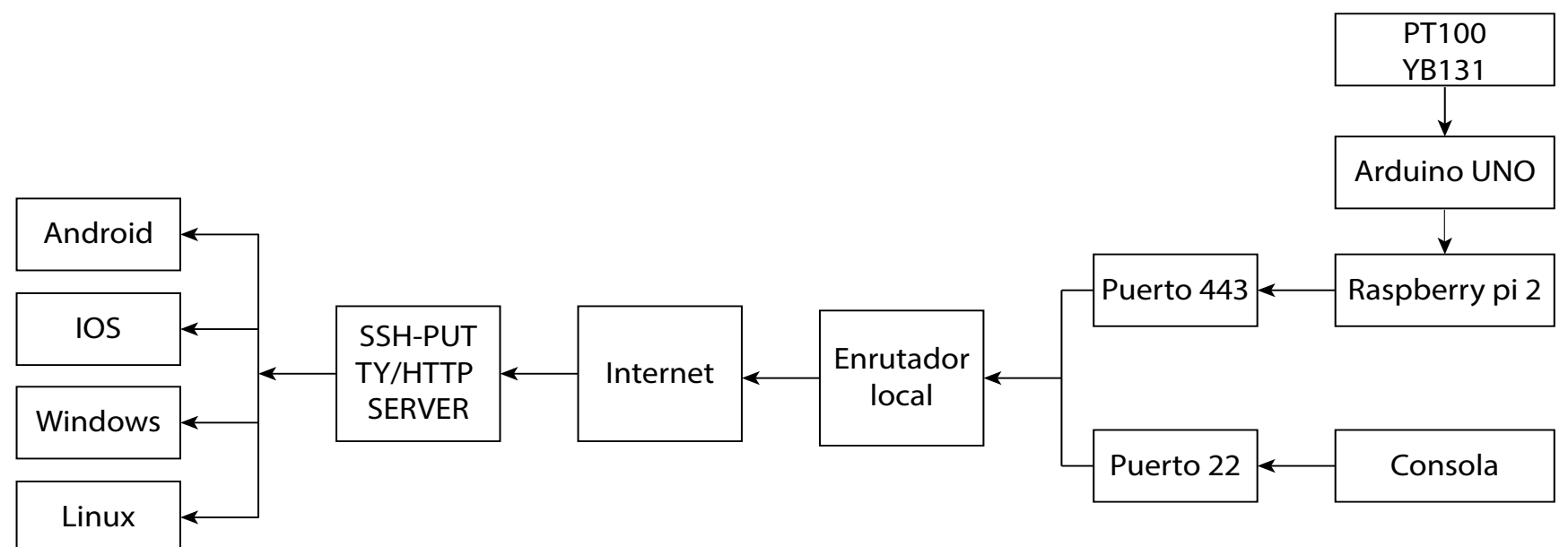

Figura 2. Diagrama de la supervisión del sistema de biodigestión anaerobia con biomasa CRB

Fuente: elaboración propia. 
Ziegler-Nichols (Åström y Hägglund, 2004; Hägglund y Åström, 2002) (tabla 2). Como el error en régimen permanente requerido ante la señal escalón fue cero, se eligió una acción de control proporcional integral $(\mathrm{PI})$, que fue programada con la librería, PID Library, de Arduino UNO para controlar la potencia suministrada por la resistencia eléctrica de $650 \mathrm{~W}$ al sistema.

Tabla 2. Parámetros del controlador Proporcional Integral según Ziegler-Nichols.

\begin{tabular}{cccc}
\hline & Kp & TI & TD \\
\hline $\mathrm{PI}$ & $0.9 \tau / \mathrm{t} \downarrow d$ & $\mathrm{t} \downarrow d / 0.3$ & \\
\hline
\end{tabular}

Fuente: adaptado de Åström y Hägglund (2004).

\section{Modelo dinámico}

Para la determinación de un modelo dinámico que explique el efecto de la temperatura sobre el fenómeno de digestión anaerobia de la biomasa CRB, se reemplazó la energía de activación y la velocidad de reacción de la ecuación de Arrhenius en términos de la entalpía de reacción y de la concentración del reactante, respectivamente (Smith, 1968) (ecuaciones (7) y (8)). Posteriormente, se establecieron las ecuaciones diferenciales del modelo matemático (ecuaciones (11), (13) y (15)) a partir de los balances de energía en el sistema (ecuaciones (9) - (16)).

$$
K(T)=A e^{-\left(\frac{\Delta H}{R T}\right)}
$$

Donde $K$ representa una constante cinética de la reacción, $D H$ la energía mínima requerida para que la reacción se lleve a cabo, $R$ representa la constante universal de los gases y $T$ es la temperatura del proceso de biodigestión; el término $A$ es una constante que antecede la exponencial y relaciona la frecuencia de las colisiones moleculares efectivas ante una determinada energía cinética.

$$
\Gamma_{B M}=-C_{B M}^{\mathrm{g}}=K(T) C_{B M}
$$

(A)

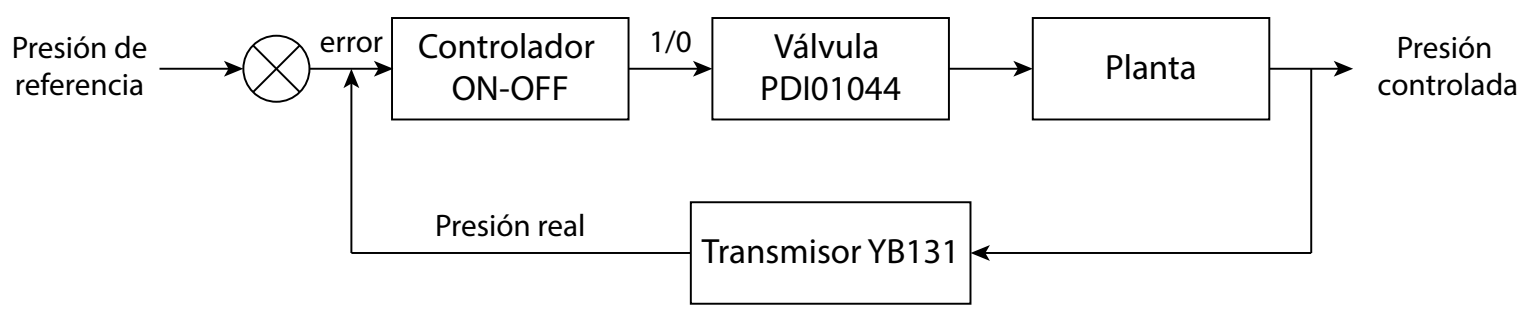

(B)

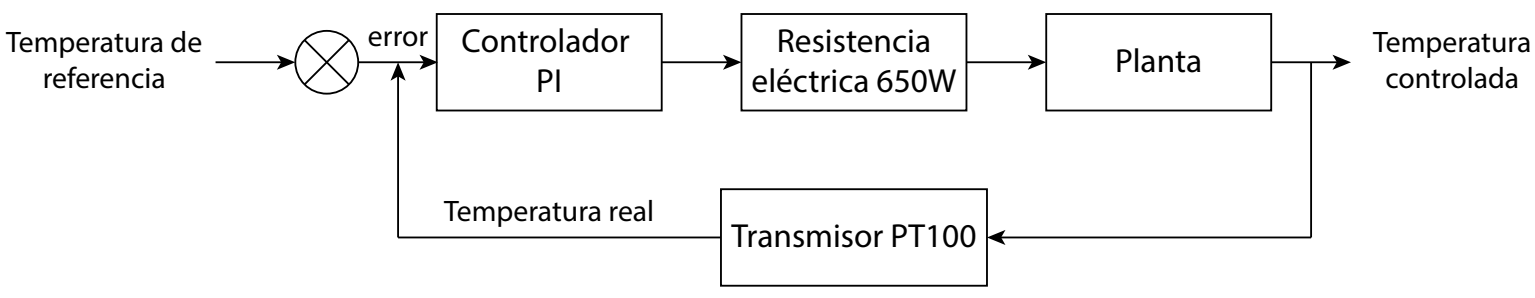

Figura 3. Esquema de control de presión y temperatura para el sistema de biodigestión anaerobia con biomasa CRB. (A). Control de presión, el controlador on-off mantuvo la presión del gas en el rango deseado. (B). Control de temperatura, el controlador (PI) garantizó la temperatura deseada en el proceso.

Fuente: elaboración propia. 


$$
\begin{gathered}
C_{B M}^{\mathrm{g}}=-K(T) C_{B} \\
C_{P}^{\mathrm{g}}=K(T) C_{B M}-K(T) C \\
\rho_{B M} C_{B M}^{*} V_{B M} T_{B M}^{\mathrm{g}}=-\mu_{i} A_{i}\left(T-T_{m}\right)-\lambda V_{B M} K(T) C_{B M}+\lambda V_{B M} K(T) C_{P} \\
T_{B M}^{\mathrm{g}}=\frac{-\mu_{i} A_{i}\left(T-T_{m}\right)-\lambda V_{B M} K(T) C_{B M}+\lambda V_{B M} K(T) C}{\rho_{B M} C_{B M} V_{B M}} \\
\rho_{M} C^{*}{ }_{M} V_{M} T_{M}^{\mathrm{g}}=-\mu_{i} A_{i}\left(T_{B M}-T_{M}\right)+\mu_{H_{2} O} A_{o}\left(T_{H_{2} O}-T_{M}\right) \\
T_{M}^{\mathrm{g}}=\frac{-\mu_{i} A_{i}\left(T_{B M}-T_{M}\right)+\mu_{H_{2} O} A_{o}\left(T_{H_{2} O}-T_{M}\right)}{\rho_{M} C_{M} V_{M}} \\
\rho_{H_{2} O} C_{H_{2} O}^{*} V_{H_{2} O}^{\mathrm{g} O} T_{H_{2} O}=-\mu_{H_{2} O} A_{H_{2} O}\left(T_{B M}-T_{M}\right)+\mu_{A M B} A_{A M B}\left(T_{H_{2} O}-T_{A M B}\right) \\
T_{H_{2} O}^{\mathrm{g} O}=\frac{-\mu_{H_{2} O} A_{H_{2} O}\left(T_{B M}-T_{M}\right)+\mu_{A M B} A_{A M B}\left(T_{H_{2} O}-T_{A M B}\right)}{\rho_{H_{2} O} C_{H_{2} O} V_{H_{2} O}}
\end{gathered}
$$

Donde los subíndices $\mathrm{BM}, \mathrm{M}, \mathrm{H}_{2} \mathrm{O}$, i y $\mathrm{AMB}$ corresponden a la biomasa, el material de las paredes del biorreactor, el agua del calentador, el interior del biorreactor y la temperatura ambiente, respectivamente; siendo $r$ la densidad, $C^{*}$ el calor especifico, $V$ un volumen asociado, $A$ un área de transferencia de calor, $m$ el coeficiente de transferencia de calor por convección, I el calor exotérmico de la reacción, G la velocidad de reacción y C la concentración.

Para la determinación del modelo de presión del gas confinado en el biorreactor se aplicó la ley de las presiones parciales (ecuaciones (17), (19) y (20)). Como la concentración del hidrogeno $(\mathrm{H} 2)$ es seis órdenes de magnitud menor, comparado con los demás subproductos generados durante la biodigestión, se despreció su magnitud y se tomó como referencia únicamente la presión del gas metano $(\mathrm{CH} 4)$ y la del dióxido de carbono $(\mathrm{CO} 2)$.

$$
P_{\text {TOTAL }}=P_{C O 2}+P_{C H 4}
$$

Dada la estequiometria de la reacción (ecuación 18), durante la combustión completa de un mol de metano $(\mathrm{CH} 4)$, se consumen 2 moles de oxígeno (O2); por lo que un mol de $\mathrm{CH} 4$ consume 64 gramos de O2; a partir de esta estequiometria, se establecieron las presiones parciales del $\mathrm{CH} 4$ y el dióxido de carbono (CO2) (ecuaciones (19) y (20)).

$$
\begin{gathered}
\mathrm{CH}_{4}+2 \mathrm{O}_{2} \rightarrow \mathrm{CO}_{2}+2 \mathrm{H}_{2} \mathrm{O} \\
P_{\mathrm{CH} 4}=C_{\mathrm{CH} 4} \frac{R T_{o p}}{64} \\
P_{\mathrm{CO} 2}=C_{\mathrm{CO} 2} R T_{o p}
\end{gathered}
$$

Por último, el modelo fue simulado mediante la herramienta computacional simulink del software MATLAB.

\section{RESULTADOS}

\section{Caracterización de la biomasa CRB}

Para caracterizar la biomasa CRB se determinó su densidad mediante análisis gravimétrico. Como se observa en la tabla 3, la densidad del CRB arrojó 
un valor de 0,99 $\pm 0,01(\mathrm{~g} / \mathrm{ml})$. Dado que esta densidad fue equivalente a la densidad del agua, se facilitó el cargue de la biomasa con una relación de proporción masa volumen de CRB y agua de 1:1. Sin embargo, la densidad no refleja la composición del CRB, por lo que fue necesario determinar su porcentaje de humedad y de materia orgánica, los cuales fueron de 71,86 $\pm 0,05 \%$ y 83,42 \pm $0,20 \%$ respectivamente; estos últimos, permitieron inferir un alto contenido de agua y de otros bioelementos que bajo un proceso de biodigestión anaerobia utilizando como biomasa al CRB pueden representar potencial en la producción de biogás.

Tabla 3. Densidad $D(\mathrm{~g} / \mathrm{ml})$, humedad $H$ y materia orgánica mo del CRB

\begin{tabular}{ccccccccc}
\hline Muestra & $\mathbf{M h}(\mathbf{g})$ & $\mathbf{V ~ ( m )}$ & Mdh $\mathbf{( g )}$ & Mic $(\mathbf{g})$ & $\mathbf{H ~ ( \% )}$ & $\mathbf{m o}(\mathbf{\%})$ & $\mathbf{D}(\mathbf{g} / \mathbf{m l})$ \\
\hline 1 & 13,16 & 13,1 & 2,82 & 0,47 & 71,8 & 83,27 & 1 \\
\hline 2 & 28,4 & 28,8 & 2,81 & 0,46 & 71,89 & 83,64 & 0,99 \\
\hline 3 & 27,22 & 27,5 & 2,81 & 0,47 & 71,89 & 83,35 & 0,99 \\
\hline & 22,93 & 23,13 & 2,82 & 0,47 & 71,86 & 83,42 & 0,99 \\
\hline
\end{tabular}

Mh representa la masa de CRB, $V$ su volumen, $M d h$ la masa deshidratada a partir de $10 \mathrm{~g}$ de CRB y Mic la masa posterior a su incineración

Fuente: elaboración propia.

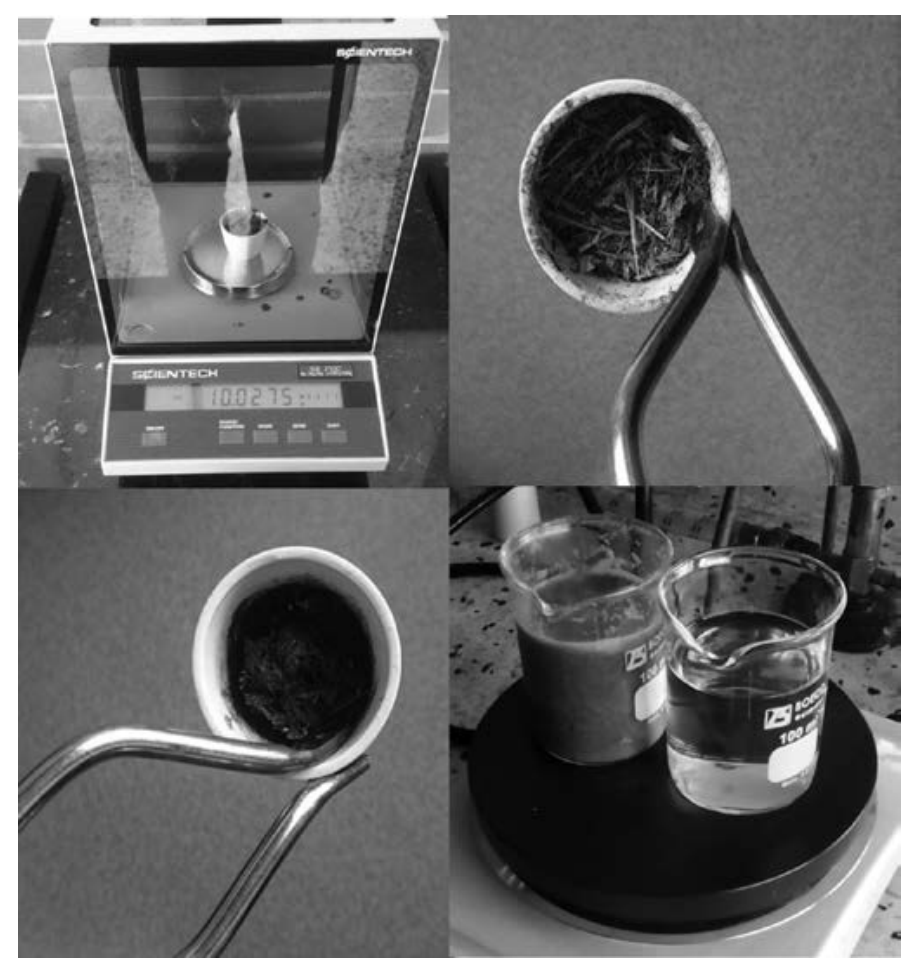

Figura 4. Ilustraciones representativas del proceso de caracterización de la biomasa CRB por gravimetría y calorimetría.

Fuente: elaboración propia. 
Tabla 4. Calor específico de la biomasa CRB C ${ }_{C R B}^{*}$

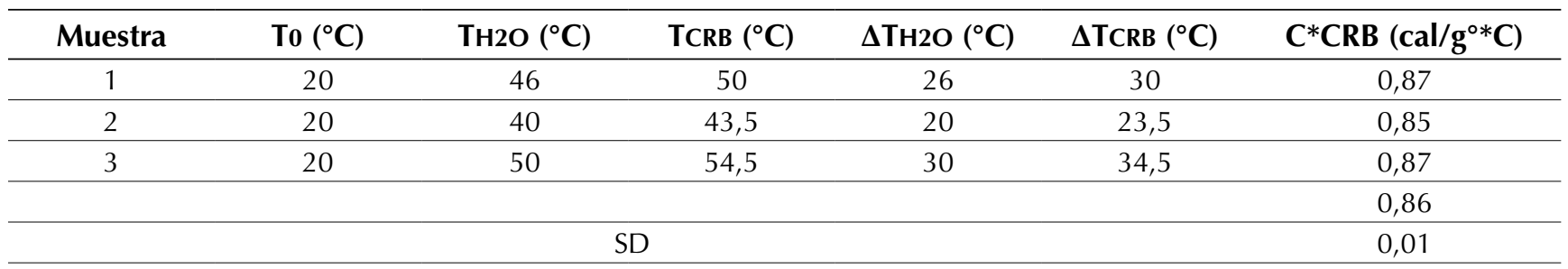

$T_{0}$ representa la temperatura ambiente, $\mathrm{T}_{\mathrm{H} 2 \mathrm{O}}$ la temperatura del agua, $\mathrm{T}_{\mathrm{CRB}}$ la temperatura de la biomasa CRB y sus respectivas variaciones respecto a la temperatura ambiente.

Fuente: elaboración propia.

\section{Presión generada en el proceso}

Con el fin de evaluar el potencial del CRB como biomasa, este fue cargado en relación 1:1 con agua destilada y sujeto a un proceso de biodigestión bajo condiciones controladas y supervisadas por 37 días (figuras 1, 2, 3). En la figura 5, se ilustran los resultados asociados al registro de la presión generada durante el proceso de biodigestión. La presión total acumulada fue de 254,99 1 1,871 (psi) con un promedio de 6,90 (psi/día). Entre los días 7 y 19 se obtuvo un promedio de gas generado de 11,38 (psi/día), que se redujo en un 35,8 \% para los días 20 a 37 (tabla 5), lo que evidencia una tendencia en fase creciente de la presión generada hasta el día 19 y un fuerte descenso en los días consecutivos (figura 5). El aumento de la presión a través del tiempo en el biorreactor es consecuencia de la generación de gas, posiblemente producto de una reacción bioquímica dependiente de microorganismos anaeróbicos, puesto que bajo condiciones aeróbicas no se generó gas. Este gas fue sujeto a combustión y generó una llama reductora de color azul, que sugiere que el biogás producto de la biodigestión posiblemente era metano.

\section{Simulaciones del modelo dinámico}

Para representar el efecto de la temperatura y la presión generada durante el fenómeno de biodigestión anaerobia para la biomasa CRB, se adaptó un modelo de cinética química en términos de los datos obtenidos de la caracterización de la biomasa y de su entalpia de reacción previamente reportados (Levenspiel y Barreiro, 2002; Smith, 1968). A partir del modelo adaptado, se aplicaron al sistema, balances de energía y la ley de las presiones parciales, estableciendo las ecuaciones diferenciales que relacionan la concentración de la biomasa CRB con el producto, las temperaturas que influyen en el sistema y la presión generada al interior del biorreactor (ecuaciones 7-20); estas ecuaciones fueron representadas en bloques y posteriormente simuladas con la herramienta Simulink del

Tabla 5. Registro de la presión del gas en los procesos de biodigestión anaerobia para CRB

\begin{tabular}{ll}
\hline \multicolumn{1}{c}{ Presión promedio(psi) } & \multicolumn{1}{c}{ Unidad (psi/día) } \\
\hline Generada entre los días 0-37 & 6,9 \\
\hline Entre los días 7-19 & 11,38 \\
\hline Entre los días 20 -37 & 7,31 \\
\hline & Unidad (psi/día) \\
\hline Acumulada entre los días 0-37 & $254,99 \pm 1.871$ \\
\hline
\end{tabular}

Fuente: elaboración propia. 


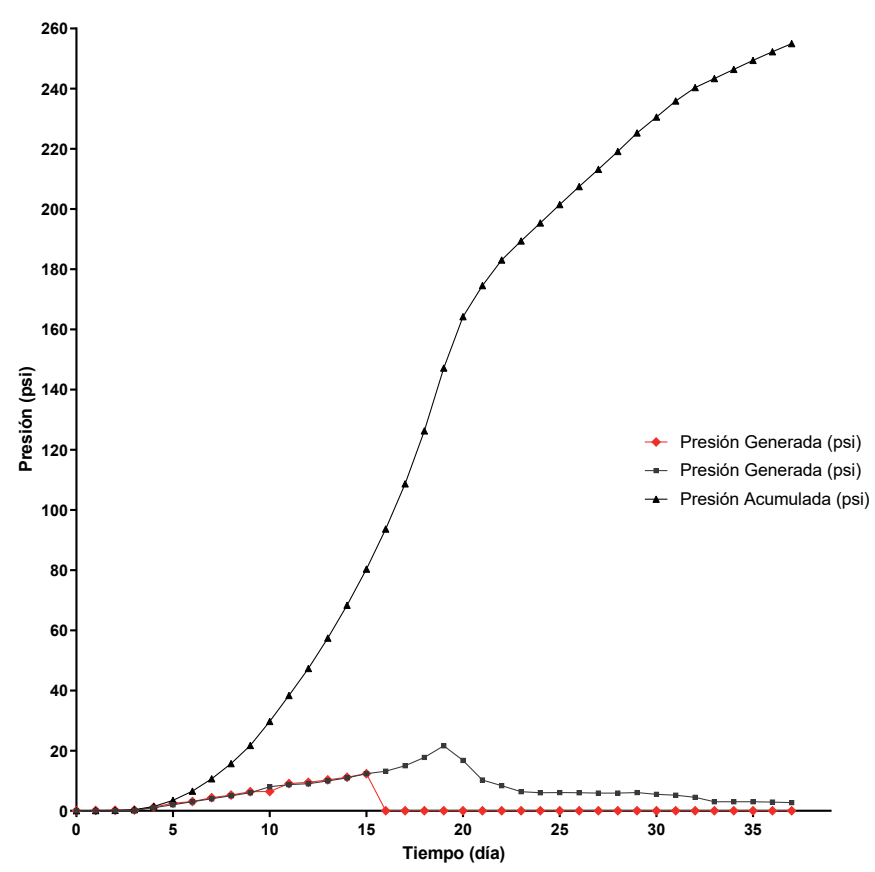

Figura 5. Presión generada y acumulada en el biorreactor a partir de la biomasa CRB

Fuente: elaboración propia.

software MATLAB. Los resultados correspondientes se presentan en la figura 6 .

Según la figura 6 (línea continua), la concentración de la biomasa CRB en función del tiempo (ecuación (9)) presenta una tendencia decreciente, mientras que la concentración del producto (ecuación (10)) crece exponencialmente (línea discontinua), hasta el punto de intersección de las dos líneas donde esta última empieza a decrecer. Con relación a las temperaturas de las paredes del reactor y de la biomasa (ecuaciones (14)-(12)), la figuras 7 y figura 8 respectivamente, muestran una tendencia críticamente amortiguada y se estabilizan en un punto de operación a $95{ }^{\circ} \mathrm{F}$. Frente a la presión generada en el biorreactor, se observa un incremento exponencial

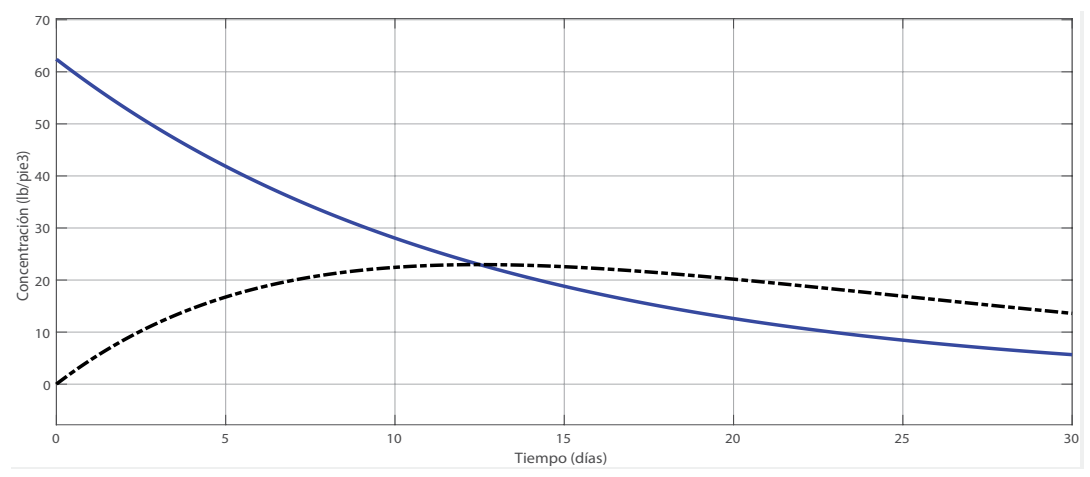

Figura 6. Concentración de CRB y su producto de biodigestión

Fuente: elaboración propia. 
dependiente de las concentraciones de la biomasa y el producto (figura 9) que se correlaciona con la tendencia registrada experimentalmente (figura 10).

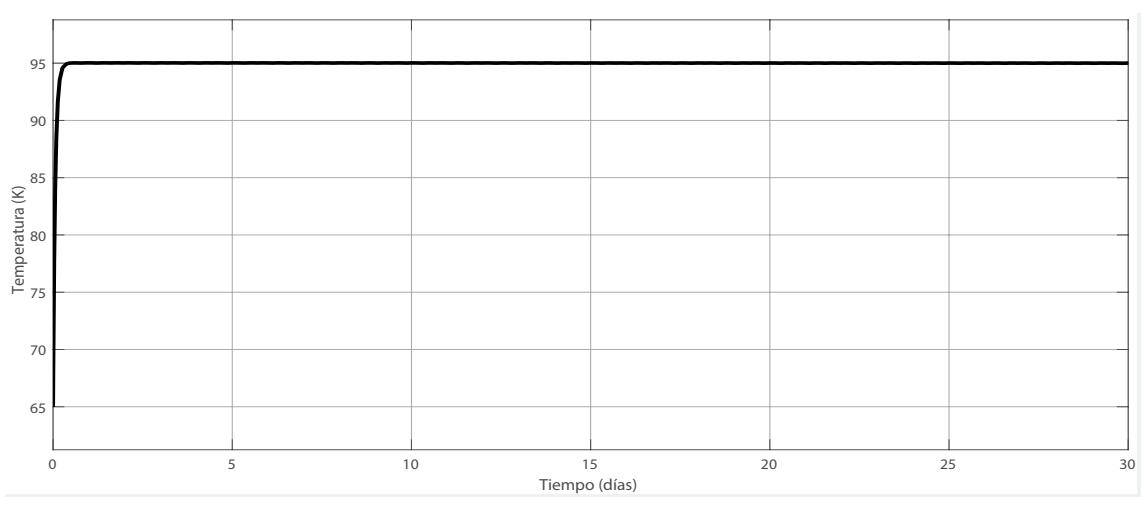

Figura 7. Temperatura de las paredes del biorreactor

Fuente: elaboración propia.

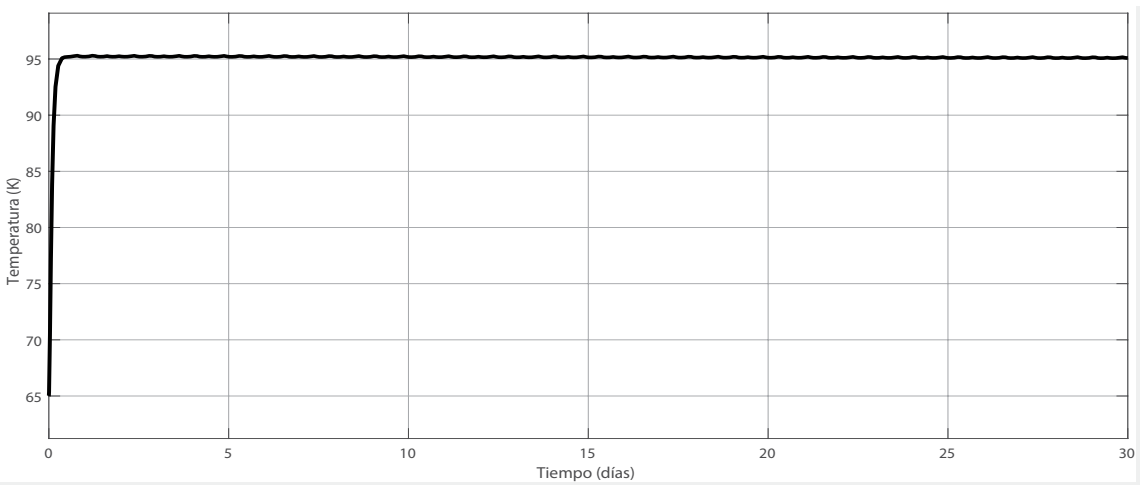

Figura 8. Temperatura del CRB

Fuente: elaboración propia.

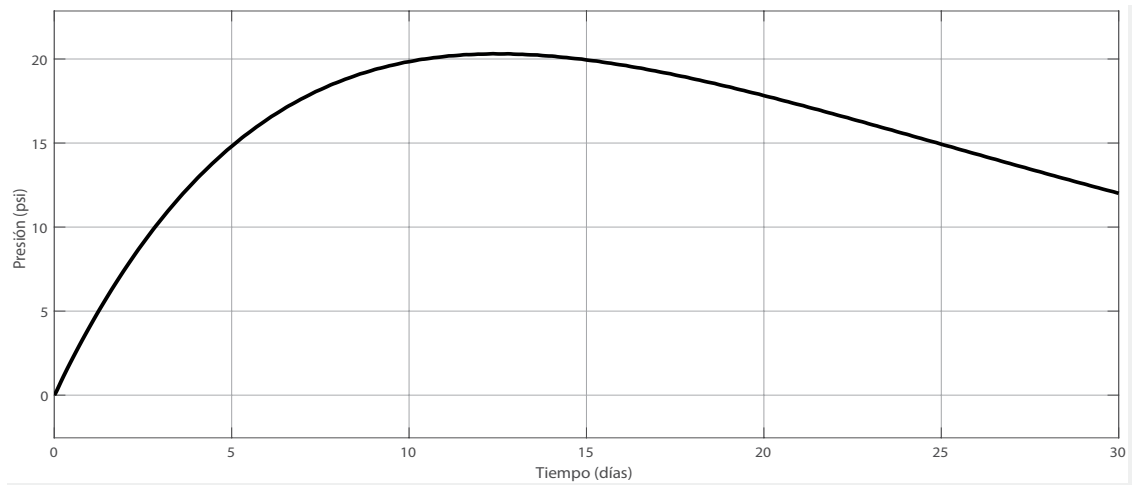

Figura 9. Presión generada en el biorreactor

Fuente: elaboración propia. 


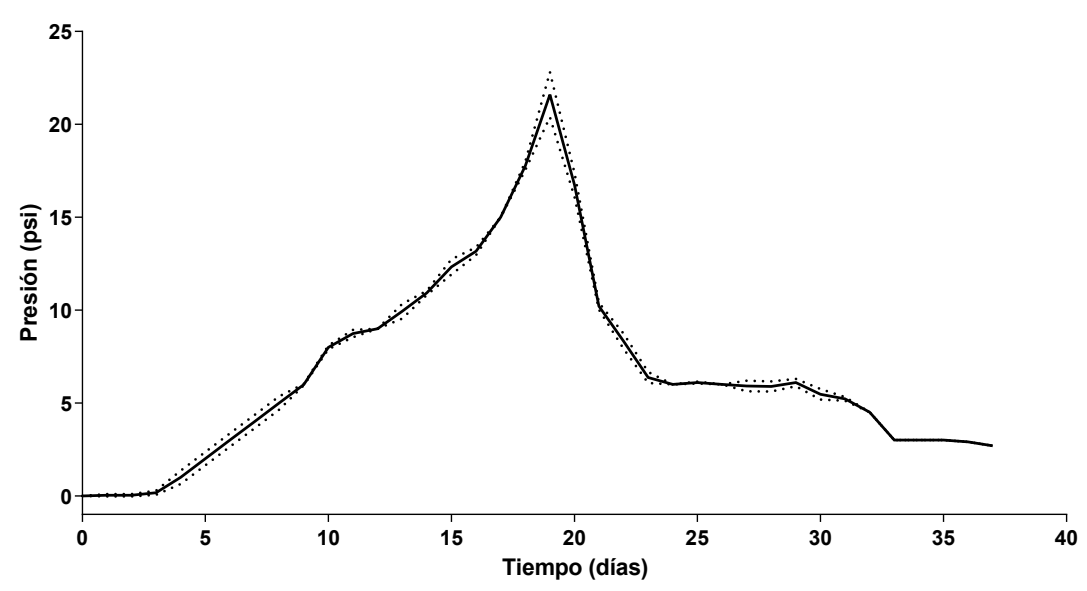

Figura 10. Correlaciona con la tendencia registrada experimentalmente

Fuente: elaboración propia.

\section{Identificación y control de la planta}

Para garantizar las condiciones de temperatura en el proceso de biodigestión anaerobia de la biomasa CRB, se realizó una identificación clásica al estímulo de la función paso sobre el sistema de calentamiento (figura 11), este ofreció en su comportamiento dinámico una función de primer orden con un retardo de 0,793 (s), una ganancia de 1,0024 , un primer tau $(\tau)$ en 4,8808 (s) y estabilidad a 27 (s) (ecuación (21)). Lo anterior sugiere que el tiempo muerto caracterizado por el retardo identificado representa el equilibrio térmico de todos los elementos que interactúan en el sistema, antes del comportamiento en fase exponencial durante el incremento de la temperatura y estabilidad del sistema.

Posteriormente se diseñó un controlador PI a través de una sintonización de parámetros con las reglas de Ziegler Nichols, ajustando el comportamiento del sistema a los valores óptimos del sobre impulso, que afectar la eficiencia en el proceso (Ramírez, 2010), mejorando la estabilidad térmica en un $40 \%$ aproximadamente (ecuación (22)).

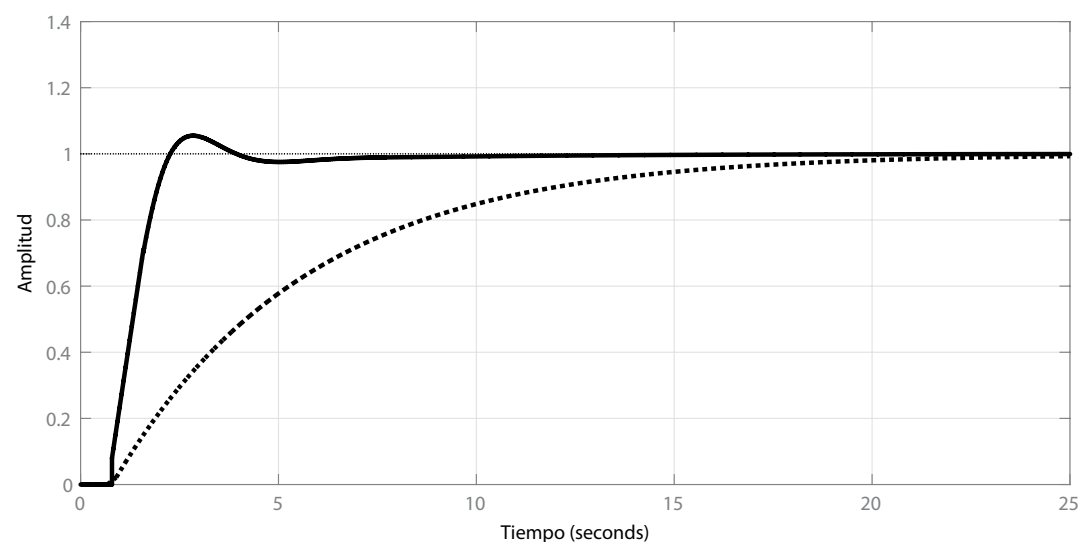

Figura 11. Identificación y control del ingenio

Fuente: elaboración propia 


$$
\begin{aligned}
& G(s)=\frac{1.0024}{4.8808 s+1} e^{0.793 s} \\
& G(s)=\frac{k}{t s+1} e^{-T s}
\end{aligned}
$$

Donde

$\mathrm{k}$ = ganancia del sistema

$\mathrm{T}=$ constante del tiempo

$\mathrm{T}=$ retardo

$$
\begin{aligned}
& C_{P I}(s)=1.66\left(1+\frac{1}{0.43 s}\right) \\
& C_{P I}(s)=K_{p}\left(1+\frac{1}{T_{1} s}\right)
\end{aligned}
$$

Donde

$\mathrm{K}_{\mathrm{p}}=$ ganancia proporcional

$\mathrm{T}_{1}=$ tiempo integral

\section{DISCUSIÓN}

La implementación de tecnologías asociadas a procesos de biodigestión anaerobia que utilicen el CRB como biomasa es limitada, en parte, a la escasa caracterización requerida para la construcción de modelos de digestión, a la restringida aplicación de sistemas de control y supervisión enfocados a mantener la estabilidad del proceso y a mejorar la eficiencia en la obtención de productos con potencial aprovechamiento energético. En este trabajo se avanzó en la caracterización parcial del CRB, y los resultados obtenidos de densidad y materia orgánica presentan concordancia con otros previamente publicados (Canepa y Olivier, 2013; Trillos, Plata, Mestre y Araujo, 2006). Frente a la composición del biogás generado, aunque la prueba de combustión permitió sugerir la presencia de metano, es necesario establecer la composición del biogás para el modelo empleado. Canepa y Olivier (2013), utilizando CRB como biomasa, reportaron un porcentaje en la producción de metano de $64 \%$.

En este trabajo también se adaptó un modelo de cinética química a la caracterización del CRB y al funcionamiento de un reactor discontinuo a escala de laboratorio; los resultados correspondientes a las simulaciones de dicho modelo se correlacionan con los datos experimentales presentados en este artículo y con los datos publicados por Smith (1968). Aunque existan modelos más descriptivos, como el presentado en 2002 por el Task Group for Mathematical Modelling of Anaerobic Digestión Processes (IWA-ADM1) (Rivera-Salvador et al., 2009), su implementación para el CRB es limitada por el desconocimiento de otros parámetros de entrada requeridos por este modelo asociados a composición bioquímica del CRB y de su producto. Finalmente, se estabilizó el proceso de biodigestión anaerobia para la biomasa CRB a los rangos de temperatura y presión previamente reportados (Ramírez, 2010), mejorando la eficiencia en la producción de biogás (datos no publicados) y se logró innovar con una supervisión web para el proceso mencionado. La proyección de esta aplicación a escala industrial requiere de evaluar la eficiencia del proceso bajo esquemas del control óptimo.

\section{CONCLUSIÓN}

Fue posible generar biogás a partir de la biomasa CRB implementando estrategias básicas de caracterización de la biomasa, supervisión y control del proceso. También se identificó un modelo matemático que se correlacionó en tendencia con el proceso de biodigestión anaeróbica para CRB en reactores tipo batch.

\section{REFERENCIAS}

Alfaro, V.M. (2001). Identificación de procesos sobreamortiguados utilizando técnicas de lazo abierto. Revista Ingeniería, 11(1), 11-25.

Álvarez, G.A. (2013). Control de temperatura y pH aplicado en biodigestores modulares de estructura flexible con reciclado de lodos a pequeña escala. Querétaro: Universidad Autónoma de Querétaro.

Arévalo, W. (2015). La biomasa: una alternativa energética proveniente de la vida misma. Editorial Unimar.

Åström, K. y Hägglund, T. (2004). Revisiting the ZieglerNichols Step Response Method for PID Control. 
Journal of Process Control, 14(6), 635-650. DOI: https://doi.org/10.1016/j.jprocont.2004.01.002

Bermúdez, J. (1988). La digestión anaerobia. Vol. 2. Murcia: Editum.

Burghardt, M.D. (1984). Ingeniería termodinámica. México: Harla.

Cadavid, C. (2001). Diseño tecnológico para el aprovechamiento de subproductos del beneficio de bovinos en el Matadero Frigorífico de Colcesar. VaIledupar, Cesar: Universidad Popular del Cesar.

Canepa, J.R.L. y Olivier, J.A.S. (2013). Degradación anaerobia del contenido gástrico ruminal bovino para la obtención de biogás, en un biodigestor tipo cúpula. Ingeniería, 1(17), 57-65.

Carrillo, L. (2004). Energía de biomasa. Edición del autor: SS Jujuy.

Denbigh, K.G. y Turner, J.C.R. (1990). Introducción a la teoría de los reactores químicos. México: Limusa.

Febres, O.A. y López, J.V. (2007). Propiedades físicas y químicas del rumen. Arch. Latinoam. Prod. Anim., 15(1), 133-140.

Fiotto, S. (2013). Modelamiento y simulación de reactores secuenciales discontinuos en un proceso de digestión anaeróbica. Bahía Blanca, Argentina: Editorial de la Universidad Tecnológica Nacional (edUTecNe).

Flórez R., D.F., Barco B., J. y Rincón P., S.L. (2016). Análisis comparativo de la carbonización de cuesco de palma de aceite en reactores de lecho fijo. Tecnura, 20(49), 45-58. DOI: https://doi.org/10.14483/ udistrital.jour.tecnura.2016.3.a03

Gómez, C.R.B. y Hernández, M.A.D. (2010). Instrumentación de un biodigestor de estiércol animal para analizar su comportamiento dinámico. Bucaramanga: Universidad Industrial de Santander.

Hägglund, T. y Åström, K.J. (2002). Revisiting the Ziegler-Nichols Tuning Rules for PI Control. Asian Journal of Control, 4(4), 364-380. DOI: https://doi. org/10.1111/j.1934-6093.2002.tb00076.x

Kreith, F., Manglik, R.M. y Bohn, M.S. (2012). Principios de transferencia de calor. México: Cengage Learning Editores.

Ledesma, L.M., Gallego, L.A. y Peláez, F.J. (2002). Situación actual de la ganadería de carne en Colombia y alternativas para impulsar su competitividad y sostenibilidad. Revista Colombiana de Ciencias Pecuarias, 15(2), 213-225.

Lettal, A., Noziere, P., Silberberg, M., Morgavi, D.P., Berger, C. y Martin, C. (2012). Rumen Microbial and Fermentation Characteristics are Affected Differently by Bacterial Probiotic Supplementation During Induced Lactic and Subacute Acidosis in Sheep. BMC Microbiology, 12, 142-153. DOI: https://doi.org/10.1186/1471-2180-12-142

Levenspiel, O. y Barreiro, G.T. (2002). Ingeniería de las reacciones químicas. Barcelona: Reverté.

Moss, A., Jouany, J.P. y Newbold, J. (2000). Methane Production by Ruminants: its Contribution to Globar Warming. Annales de Zootechnie, 49(3), 231-253. DOI: https://doi.org/10.1051/animres:2000119

Pedreira, M.S., Oliveira, S.G., Primavesi, O., Lima, M.A., Shiraishi, R.T. y Berchielli, T.T. (2013). Methane Emissions and Estimates of Ruminal Fermentation Parameters in Beef Cattle fed Different Dietary Concentrate Levels. Revista Brasileira de Zootecnia, 42(8), 592-598. DOI: https://doi.org/10.1590/ S1516-35982013000800009

Quiñones, C.Q.C. (2006). Energías alternativas. "Solución para el desarrollo sustentable". Chile: Adnuma. Ramírez, I.F. (2010). Emisiones de metano generadas por excretas de animales de granja y contenido ruminal de bovino. (Tesis doctoral). Colegio de Postgraduados, México.

Ríos, M. y Ramírez, L. (2012). Aprovechamiento del contenido ruminal bovino para ceba cunicola, como estrategia para diezmar la contaminación generada por el matadero en San Alberto. Prospectiva, 10(2), 56-63. DOI: https://doi.org/10.15665/ rp.v10i2.234

Rivera-Salvador, V., Aranda-Barradas, J. S., Espinosa-Solares, T., Robles-Martínez, F. y Toledo, J.U. (2009). El modelo de digestión anaeróbica IWA-ADM1: una revisión de su evolución. Ingeniería Agrícola y Biosistemas, 1(2), 109-118. DOI: https://doi. org/10.5154/r.inagbi.2010.01.001

Smith, J.M. (1968). Ingeniería de la Cinética Química. Trad. A.E. Gómez. México: Compañía Editorial Continental. 
Tardáguila, R.D. (2008). La biomasa como recurso energético. En P. Ramos (ed.), Energías y cambio climático (pp. 31-63). Salamanca, España: Ediciones Universidad de Salamanca.

Torge, W. (1989). Fundamentos, métodos y problemas de la gravimetría. En $V$ Curso de Geodesia Superior (pp. 1-83). Madrid: Instituto de Astronomía y Geodesia.

Torres, K.R.M. y Manga, Y.M. (2013). Tratamiento de aguas residuales a partir de digestión anaerobia. Ingeniator, 4(6), 118-135. Universidad de San Buenaventura seccional Cartagena.

Trillos, G.L., Plata, O.L., Mestre, A.T. y Araujo, G.A. (2006). Análisis fisicoquímicos de los contenidos ruminales frescos y ensilados de bovinos sacrificados en el Valle del Cesar. Valledupar, Colombia: Facultad de Ingenierías, Programa de Agroindustria,
Universidad Popular del Cesar. Recuperado de https://www.engormix.com/ganaderia-carne/articulos/analisis-fisico-quimicos-contenidos-t26583.htm

Uicab-Brito, L. y Castro, C.S. (2003). Uso del contenido ruminal y algunos residuos de la industria cárnica en la elaboración de composta. Tropical and Subtropical Agroecosystems, 2(2), 45-63.

Vargas-Soplín, A.d.J. y Ramírez-Candia, J.M. (2017). Determinación de polos de generación distribuida a partir de biomasa residual agrícola en la región Madre de Dios, Perú. Tecnura, 21(53), 61-77.

Vincent, T. (1997). Digestión anaerobia, bases cinéticas y microbiológicas. Barcelona: Universidad Autónoma de Barcelona.

Vogel, A.I. (1960). Química analítica cuantitativa: teoría y práctica. Buenos Aires, Argentina: Editorial Kapelusz. 